\title{
Using Datacube Technology for In-Situ-Enhanced Precision Farming
}

\author{
Peter Baumann ${ }^{1}$, Katrin Kohler ${ }^{2}$, Vlad Merticariu ${ }^{1}$, Ismoil Isroilov ${ }^{2}$ \\ ${ }^{1}$ rasdaman $\mathrm{GmbH}$, Germany \\ ${ }^{2}$ Spatial Business Integration GmbH, Germany \\ \{baumann,merticariu\}@rasdaman.com \\ $\{$ k.kohler,i.isroilov\}@spatial-business-integration.com
}

\begin{abstract}
Goal of the BigPicture project is to enhance satellite-based information with empirical in-situ ground-truthing. In this approach, the symptoms detected from remote sensing data are a mere starting point where additional data - like long-term time series, location of unusually growing areas, and weather conditions - get mixed in to ultimately obtain recommendations on soil treatments and applications of seeds, fertilizers and plant protection products.

In this case study we present BigPicture as a practical, non-trivial application of the emerging datacube paradigm It shows a servicecentric approach using a high-level standardized query language, the OGC Web Coverage Processing Service (WCPS), rather than low-level procedural programming approaches.
\end{abstract}

\section{CCS CONCEPTS}

- Information systems $\rightarrow$ Data management systems; Database management system engines; Parallel and distributed DBMSs; Information systems applications; Information systems applications; Geographic information systems

\section{KEYWORDS}

Precision farming, ground truthing, datacube, analytics, rasdaman, OGC WCPS

\section{ACM Reference format:}

Peter Baumann, Katrin Kohler, Vlad Merticariu, Ismoil Isroilov. 2018. Using Datacube Technology for In-Situ-Enhanced Precision Farming. In Proceedings of ACM SIGSPATIAL conference (SIGSPATIAL'18). ACM, New York, NY, USA, 7 pages. https://doi.org/10.1145/3282834.3282840

\section{Introduction}

Permission to make digital or hard copies of part or all of this work for personal or classroom use is granted without fee provided that copies are not made or distributed for profit or commercial advantage and that copies bear this notice and the full citation on the first page. Copyrights for third-party components of this work must be honored. For all other uses, contact the owner/author(s). BigSpatial 2018, November 6, 2018, Seattle, WA, USA

(C) 2018 Copyright held by the owner/author(s). ISBN 978-1-4503-6041-8/18/11. https://doi.org/10.1145/3282834.3282840
Satellite images show the response of plants to various impact factors affecting growth, vitality, health, and ultimately yield potential. However, it is not entirely straightforward to derive guidance to the farmers from satellite imagery. Goal of the BigPicture project [23] is to enhance satellite-based information with empirical in-situ ground-truthing. In this approach, the symptoms detected from remote sensing data are a mere starting point where additional data - like long-term time series, location of unusually growing areas, and weather conditions - get mixed in to ultimately obtain recommendations on soil treatments and applications of seeds, fertilizers and plant protection products.

Obviously, such an endeavor needs to rely on a variety of data which together constitute "Big Data" given their volume, variety, and complexity in analytics. At the heart is a timeseries of satellite imagery, effectively forming a 3-D x/y/t datacube, complemented by a weather datacube.

In this case study we present a practical, non-trivial application of the emerging datacube paradigm; in particular, it shows analytics functionality, rather than low-level programming approaches. Concretely, the system architecture centers around the rasdaman datacube analytics engine.

The remainder of this paper is organized as follows. In the next section we introduce the use case. The concept of datacubes, as well as a rasdaman overview, are presented in Section 3 and 4, upon which the technical approach is unfolded in Section 5. A performance analysis is given in Section 6 , followed by a review of related work in Section 7. Finally, conclusions are presented in Section 8 .

\section{Enhancing Satellite-Based Precision Farming with In-Situ Ground Truthing}

The relationships between symptoms and causes in crop canopies are investigated in a variety of fields in different growing areas in Germany during three cultivation seasons. Figure 1 shows the locations of the 80 farmers who provided approximately 800 fields for data collection in 2017. In addition, 50 sales representatives from the agrochemical industry are involved in the project.

Up to six satellite image based Biomass maps are delivered for fields cultivated with winter wheat, winter rape seed and corn in the time period from March to August. Farmers are interviewed 
about the causes of the plant's symptoms, the treatments and the field histories. This information is used to define the relationships and serves as reference for the analyses of unknown fields. In this project, "Big Data", in the sense of voluminous data, are mainly derived from raster data such as satellite images and their products, satellite image time series, elevation models, etc. In order to be flexible and scalable, the rasdaman array database is used, providing management as well as querying of multidimensional raster data.

An online portal has been set up for uploading the field borders and for delivering the satellite image-based Biomass maps to the farmers. A so-called field pattern occurs if the biomass grows better or worse in a sub-area compared to the rest of the field. Figures 2a-c show field patterns in the example of a field located in a highly fertile cultivation region in Germany, the Magdeburger Boerde. The field was cultivated with winter wheat in 2015 and winter oilseed rape in 2016. The yellowish color in the satellite image maps $2 a$ ) and $2 b$ ) identifies zones with lower biomass growth.

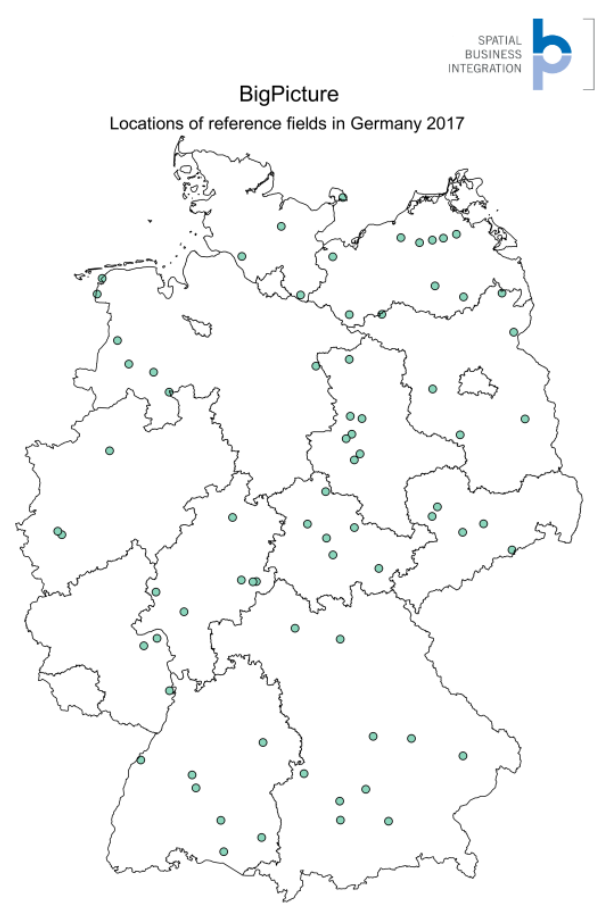

Figure 1: Locations of German reference fields in 2017

If the same field patterns appear every year, regardless of cultivated crop, treatments and weather conditions, a dominant permanent influence of soil properties on plant growth can be deduced. In order to separate such long-term from current influences, Field Potential Maps were calculated for all arable land in the test areas. For this purpose, several satellite images taken in, at least, three to five years were matched. Figure $2 \mathrm{c}$ shows an example of the Field Potential Map, which clearly identifies long-term stable soil properties. The brown, orange and yellowish areas localize zones with lower loess cover, due to underlying gravel and sand layers.

Current causes of field patterns can be frost, drought and hail damage, disease occurrence or nutrient deficiency. As one scenario, the detection of frost damage was defined in the project. The area and severity of frost damage in field crops determine the loss of yield and, hence, require the decision on re-seeding.

The request was based on the assumption that frost damage occurs in a winter crop in Germany, if the biomass in March is smaller than in November or December and has not recovered by April or has continued to decline. With the same request, it was clarified whether there were frost days over wintertime with simultaneous lack of snow cover. In the cultivation region, having a size of $37,000 \mathrm{~km}^{2}$ and about 400,000 fields, this condition was fulfilled on an area of 1,440 ha (3.558 acres), which were assigned to the affected fields. a)

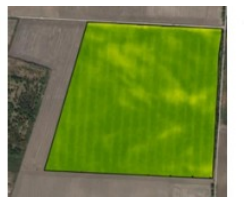

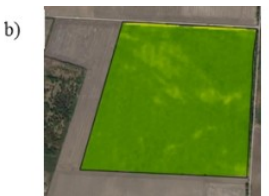

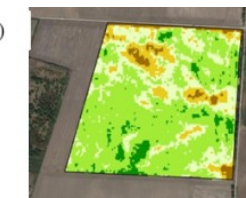

Figure 2: Satellite-image-based Biomass maps - a) winter wheat on 2015-04-24, b) winter oilseed rape on 2016-06-05, c) multi-year Field Potential Map (source: Landwirtschaftliche Betriebsgemeinschaft GbR Groß-Germersleben; includes material (2018 Planet Labs Germany GmbH, Google Earth, Spatial Business Integration GmbH)

\section{Datacubes}

A datacube, in the first place, is given by a massive multidimensional array, also called "raster data" or "gridded data"; "massive" entails that we talk about sizes significantly beyond the main memory resources of the server hardware. Data values, all of the same data type, sit at grid points (cells) as defined by the $\mathrm{n}$ axes of the n-dimensional datacube. Coordinates along these axes allow addressing data values unambiguously.

An n-dimensional grid is characterized by the fact that each inner grid point has exactly two neighbors along each direction; border grid points have just one (see Fig.3). Point clouds, e.g., are not grids.

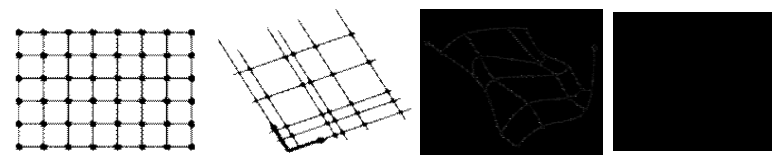

Figure 3: Sample regular and irregular grids [17]

In order to represent all this information, a datacube contains not only the bare array, but also ornamenting metadata adding all the relevant information about space and time semantics (i.e., Coordinate Reference systems, CRSs), etc.

Actually, the datacube concept constitutes nothing but a logical model for rapid application development; it does not anticipate 
any storage model, thereby hiding details like tiling, data format, serialization, possible distribution, etc. The OGC Coverage Implementation Schema (CIS) in its current version 1.1 offers a concise, yet flexible structure.

However, this is only the data structure. From a service perspective, datacubes just as much need to offer a streamlined set of operations coming as convenient APIs. For geo datacubes, an interoperable role model is given by the OGC Web Coverage Service (WCS) standard [19] with its geo datacube analytics language, Web Coverage Processing Service (WCPS) [16]. An overview on the OGC datacube standards is provided at [18].

These principles have been summarized in the Datacube Manifesto [4] and extended by The Six Faces of The Datacube [22].

\section{4 rasdaman}

The rasdaman Array Database enables management of massive multi-dimensional arrays, together with declarative array operators which are heavily optimized and parallelized on server side. A separate layer adds geo semantics, such as knowledge about regular and irregular grids and coordinates, by implementing the OGC Web service interfaces. The open-source rasdaman engine is OGC datacube reference implementation. We briefly describe the system and refer to [20] for details.

Array Storage. For storage, arrays get partitioned ("tiled") into sub-arrays [7] which are maintained either in a conventional database (such as PostgreSQL) or its own persistent store directly in any kind of file system. Additionally, rasdaman can tap into "external" files not under its control by just registering these files, rather than importing them. Internal tiling of archive files (such as available with TIFF and NetCDF, for example) can be exploited for fine-grain reading.

A core concept of array storage in rasdaman is partitioning or tiling. Arrays are split into sub-arrays called tiles to achieve fast access. Tiling policy is a tuning parameter for adjusting partitions to any given query workload, measured or anticipated. As this mechanism turned out very powerful for users, its generality has been cast into a few strategies available to data designers (Fig. 4).

Array Processing. As shown in Fig. 5, the rasdaman server processes ("rasserver") constitute the central workhorses. It can access data from various sources for multi-parallel, distributed processing. The rasdaman engine has been crafted from scratch, optimizing every single component for array processing. A series of highly effective optimizations is applied routinely to queries, including:

- Query rewriting to find more efficient expressions of the same query; currently about 150 rewriting rules are implemented.

- Query result caching serves to keep complete or partial query results in (shared) memory for reuse by subsequent queries; in particular, geographic or temporal overlap can be exploited.

- $\quad$ Array joins [3] with optimized tile loading so as to minimize multiple loads when combining two arrays. This is not only effective in a local situation, but also when tiles have to be transported between compute nodes or even data centers in case of a distributed join.

After query analysis and optimization, the system fetches only the tiles required for answering the given query. Subsequent processing is highly parallelized. Locally, it assigns tiles to different CPUs / GPUs and threads where each one can take over individual operations, exploiting the particular strengths of the processors (such as SIMD proecsing). In a cluster or cloud, queries are split and parallelized across the nodes (Fig. 6). The same mechanism is also used for distributing processing across data centers, where data transport becomes a particular issue. To maximize efficiency, rasdaman currently optimizes splitting along two criteria: First, send queries to where the data sit, according to the Big Data principle of "ship code to data". Second, generate subqueries that process as much as ever possible locally, minimizing the amount of data to be transported between nodes.

This way, single queries have been split successfully across more than a thousand Amazon cloud nodes [6].
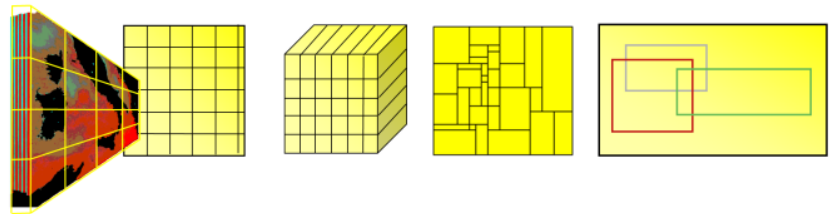

Figure 4: Sample tiling rasdaman strategies supported (source: rasdaman)

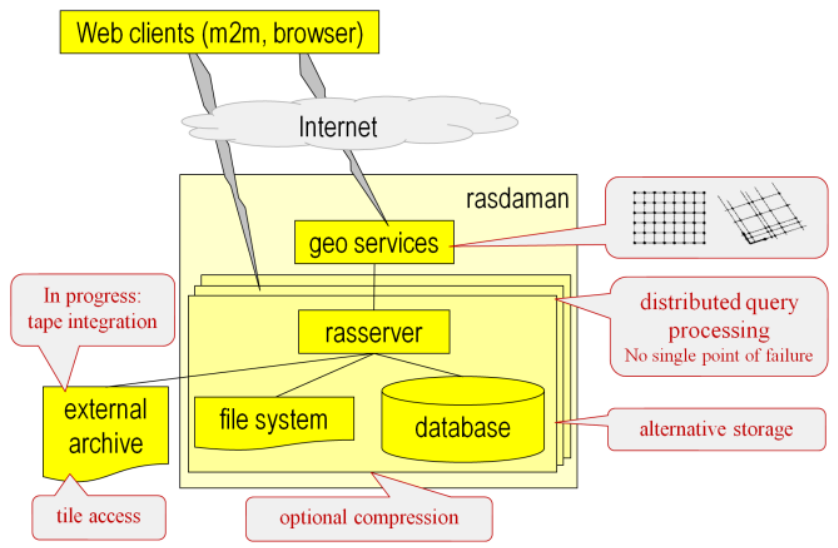

Figure 5: rasdaman overall architecture (source: rasdaman)

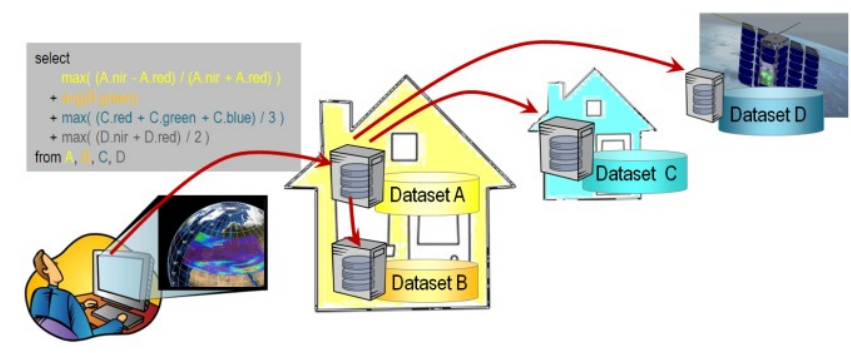

Figure 6: rasdaman query splitting (source: rasdaman) 


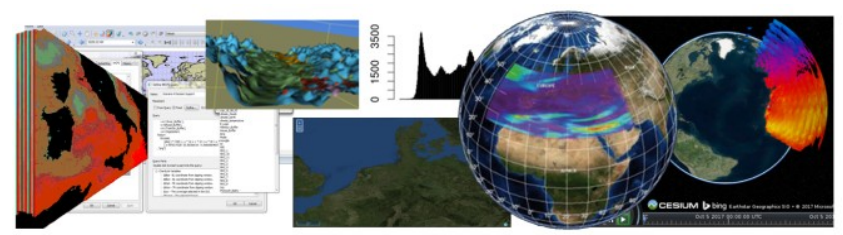

Figure 7: Selection of clients accessing rasdaman-powered datacube services (from left to right): DLR 3D client; Envitia Chartlink; WebGL; OpenLayers; python; NASA WorldWind; Cesium (source: rasdaman/EarthServer)

Tool integration. Via the OGC WMS, WCS, and WCPS standards protocols rasdaman supports major GIS Web and programmatic clients, with more under development (Fig. 6). Among these are OpenLayers and Leaflet, QGIS and ArcGIS, NASA WorldWind and Cesium, $\mathrm{C}++$ and Java, as well as python and $\mathrm{R}$.

\section{Precision Farming Scenario}

The requirement is to determine all pixels in the area of interest that simultaneously satisfy the following conditions:

1. The NDVI (Normalized Difference Vegetation Inex) at the end of March is lower than the NDVI at end of December.

2. The NDVI at the end of March is equal to the NDVI at the end of April.

3. The temperature at the Lat,/Long coordinates of the pixel satisfying conditions 1 and 2 has been negative at least once during winter.

The datacubes used were the following:

- Sat_red and Sat_nir: red and near infra-red channels of satellite imagery in the area of interest. These are 3D datacubes, with spatial extent $275985 \mathrm{~m}$ to $582315 \mathrm{~m}$ in Easting, $5292285 \mathrm{~m}$ to $5691615 \mathrm{~m}$ in Northing (crs is EPSG 32632), and temporal extent "2016-1229T00:00:00.000Z" to "2017-04-20T00:00:00.000Z"(crs is OGC AnsiDate, a time axis understanding ISO 8610 date and time syntax).

- Temp2m: 3D datacube of Kelvin temperature values, with spatial extent 47 degrees to 55 degrees in Latitude, 6 to 15 degrees in Longitude (CRS is EPSG 4326), and "2016-11-01T00:00:00.000Z" to "2018-0131T18:00:00.000Z" in time (CRS is OGC AnsiDate).

The service used to query and combine the datacubes was WCPS [16], an OGC standard web service for performing on-thefly analytics on datacubes. The user writes a query and sends it to the server hosting the datacube, the server evaluates it and sends the result back to the user.

Computing the NDVI at a certain date is achieved in WCPS by the following query (Q1):

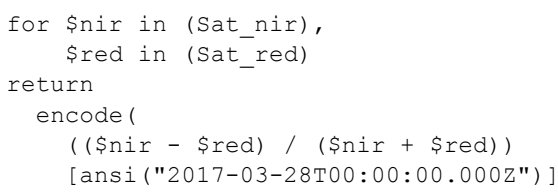

, "tiff")

The query performs a slicing operation at time "2017-0328T00:00:00.000Z", obtaining the corresponding 2D subsets of the cubes, then combines them to obtain the result: a tiff image where each pixel is the ratio between the difference and the sum of the nir and red channels. By comparing the NDVI values at the dates of interest, we can already find the pixels satisfying conditions 1 and $2(\mathrm{Q} 2)$ :

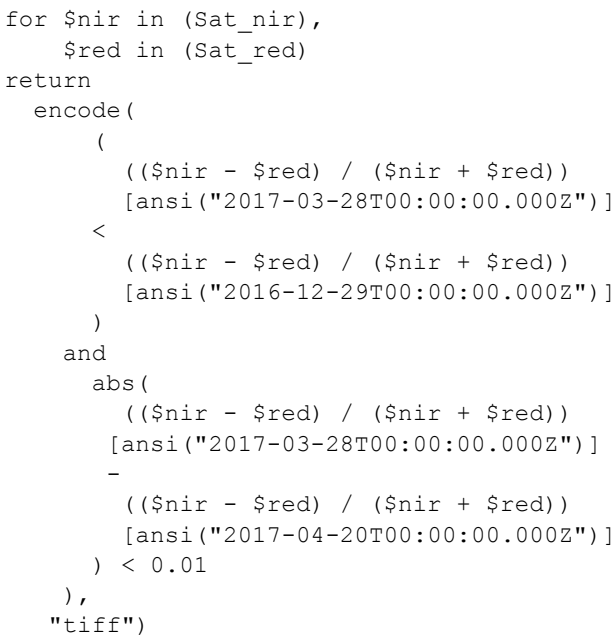

In the first part (up to the and operator), the query computes a Boolean mask identifying the pixels satisfying condition 1 . In the second part, the query addresses condition 2 . Since NDVI consists of floating point values between -1 and +1 , an exact equality comparison is very susceptible to numeric effects during computation; to avoid artificially introduced mismatches, an interval comparison with a radius of 0.01 was chosen, expressed as abs $(\ldots)<0$. 1. Finally, the and operation combines the two masks into the final result.

In order address condition 3., the query has to check, for each True pixel in the mask, whether the temperature has been negative at least once during winter at that particular location. There are several valid approaches for doing that in WCPS. For the reader's convenience, we are presenting one that reduces the query sizes.

In a preparatory step, we are condensing the temperatures cube into a single image where each pixel is the minimum recorded temperature during winter at its Lat, Long coordinates. Furthermore, we are performing a reprojection operation to bring the minimum temperatures image into the same coordinate system as the satellite data $(\mathrm{Q} 3)$ :

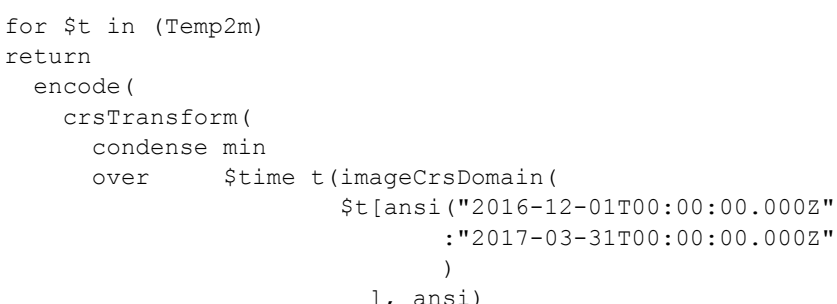




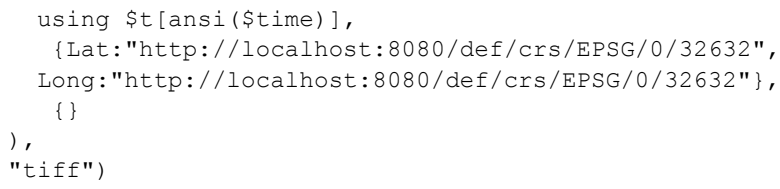

The result was re-ingested into the rasdaman database and made available in WCPS under the name MinimumWinterTemp. With it, we can build the final query (Q4):

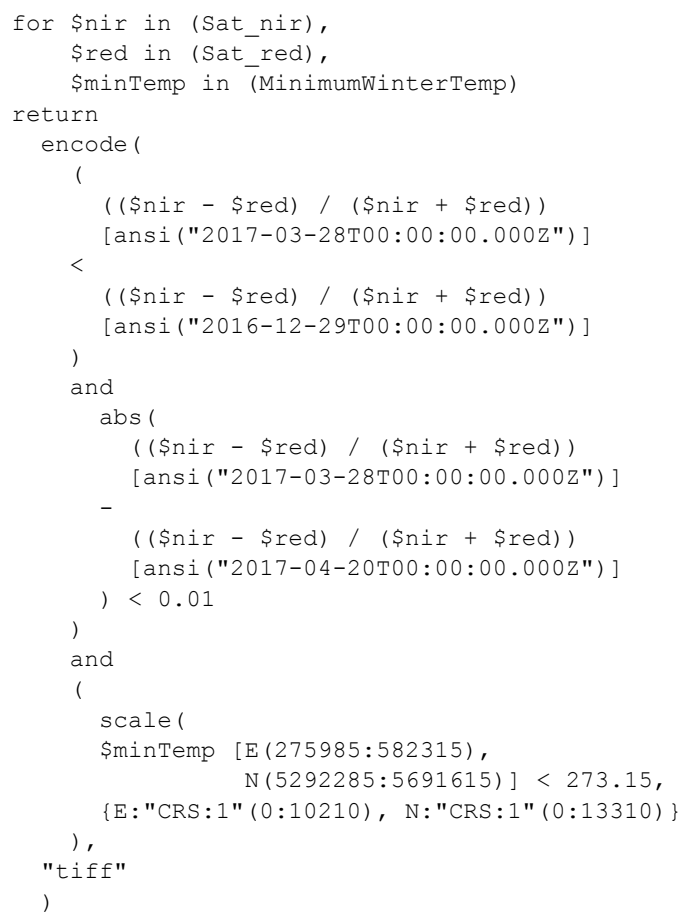

The query adds a further and operation that checks, for each pixel that the minimum temperature recorded at that location was less than $273.15 \mathrm{~K}$ (i.e., $0{ }^{\circ} \mathrm{C}$ ), thus fulfilling the requirement. Because the satellite and temperature datasets have different resolutions, a scale operation is applied before applying the masks to obtain the final result.

\section{Evaluation}

Performance of queries Q1 to Q4 depends mainly on the size of the area on which they are applied. In the form described in Section 4, the queries work on the entire area of interest in one step, producing a single result containing information about all the fields in the area. While this saves overhead, as opposed to running a query for each field individually, we wanted to measure whether this can still be done in real time.

The measurements were performed in a virtual machine served by 4 cores of an Intel Xeon E5-2699 CPU, with a $2.3 \mathrm{GHz}$ clock speed, 32 GB of RAM and a standard hard disk with a sequential read speed of about $100 \mathrm{MB} / \mathrm{sec}$.

We measured the execution time of each query up to 10 times and chose the median execution time after excluding outliers as result. All query runs were done on a cold database, with no caching. The table below shows the execution times, together with the amount of data touched by every query.

\begin{tabular}{|c|c|c|c|c|}
\hline & Q1 & Q2 & Q3 & Q4 \\
\hline Data (MB) & 1087 & 1087 & 39 & 1126 \\
\hline Ex. Time (s) & 7.94 & 8.04 & 0.22 & 8.42 \\
\hline
\end{tabular}

Table 1: Test queries with data volume and runtimes

The measurements show that the analysis can be confidently run in real time, using a single query for the entire area of interest. Q1, Q2 and Q4 run on very similar amounts of data but involve significantly different amounts of processing. However, the execution times are very close, which makes us believe that in the current set-up the main bottleneck is the disk.

\section{Related Work}

Image processing tools and libraries such as MatLab [9] have been around since long, some of them even capable of handling multi-dimensional arrays. However, all these tools have originally been developed for desktop applications; hence, they (i) are basically limited to main-memory size objects and cannot efficiently handle data orders of magnitude beyond that and (ii) are not naturally architected as service backends handling multiple simultaneous requests appropriately.

According to RDA [21] the range of technologies supporting "Big Arrays" can be classified into Array Databases, programming interfaces, and MapReduce type systems. We briefly discuss each category in turn:

- Programming interfaces encompasss command-line tools and libraries which, when getting embedded in some server, servers accepting some procedural language. regular procedural code is submitted to the server. Examples include Google Earth Engine [8] as well as Open Data Cube [12] with its python API. Further, this category includes libraries, like xarray [14] and Ophidia [15].

Following database community common sense, procedural APIs suffer from several disadvantages as compared to query-based systems like rasdaman:

- Writing requests to the server requires substantial programming skills in the particular language chosen, and the code is difficult to debug as it requires server data access. For example, declarative query language statements are typically very few lines whereas the corresponding program code tends to stretch over several pages.

- Procedural code is hard to optimize in the server, as compared to declarative query languages.

- Procedural code opens doors widely for denial-ofservice attacks whereas SQL, the rasdaman language, rasql, and OGC WCPS are known to be "safe in evaluation".

- While languages like python and Java support arrays in main memory (enhanced by libraries like xarray) 
there is a lack of disk (and tape) storage management with automated partition management, distributed array join handling [3], etc.

- MapReduce style systems, extending Hadoop or Spark with specific array functionality. Here we find representatives like GeoTrellis [13].

Generally, the MapReduce paradigm comes with two essential shortcomings: First, it is unaware of the array structure; the common main-memory oriented libraries which are added in often cannot achieve a globalized array-aware distributed storage management. Second, the rigid parallelization schema "map; reduce; repeat" cannot leverage all parallelization potential, as we will show in the demo.

- Array Database Systems offer declarative query support for multi-dimensional arrays. Representatives include rasdaman, PostGIS Raster [10] and SciDB [11]. Through their high-level query languages in combination with strong automatic optimization and parallelization capabilities this category represents the "gold standard". The rasdaman system stands out through its performance, scalability, and standards support (see RDA report below).

The above considerations get reinforced by the deep functionality, architecture, and performance comparison available from the Research Data Alliance (RDA) report [21]. Fig. 8, taken from this report, shows the result of running 22 test queries covering a broad range of multi-dimensional functionality. As it turns out, rasdaman can be 300x faster than other array tools.

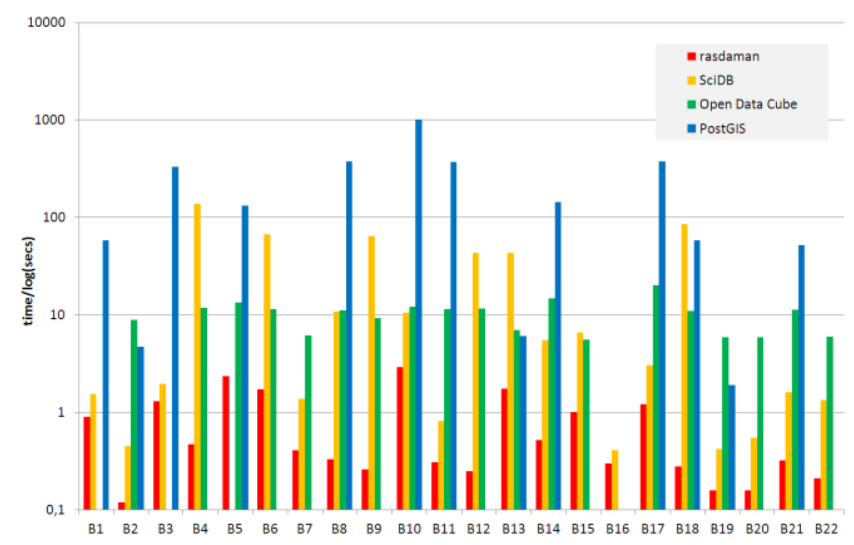

Figure 8: Performance comparison of four datacube servers based on 22 criteria (note the logarithmic time axis) [21]

\section{Conclusion}

The system presented in this contribution will be published as a portal accessible via both fixed-position and (with primary focus) mobile end devices such that farmers get pointed at relevant phenomena discovered in satellite imagery, together with suggestions for explanation and treatment. At the same time, such derived maps can be encoded in the ISOBUS format for direct feed into agricultural machinery.
We believe that this approach is superior to services which stop at providing purely visual, un-interpreted satellite information. Use of the datacube engine, rasdaman, has shown several advantages. On the one hand, design was agile and allowed rapid prototyping - which also is expected to ease future adaptations, modifications, and enhancements. On the other hand, the performance achieved on both simple and complex queries enables rich functionality in real-time and without any programming (such as python), heralding a new generation of service quality for farmers.

\section{ACKNOWLEDGMENT}

This work is partially supported by German BMEL (Ministry of Food and Agriculture).

\section{REFERENCES}

[1] P. Baumann, A.P. Rossi, B. Bell, O. Clements, B. Evans, H. Hoenig, P. Hogan, G.1 Kakaletris, P. Koltsida, S. Mantovani, R. Marco Figuera, V. Merticariu, D Misev, B. Pham Huu, S. Siemen, J. Wagemann, Fostering Cross-Disciplinary Earth Science Through Datacube Analytics. In. P.P. Mathieu, C. Aubrecht (eds.): Earth Observation Open Science and Innovation - Changing the World One Pixel at a Time, International Space Science Institute (ISSI), 2017 (in print)

[2] P. Baumann, D. Misev, V. Merticariu, B.P. Huu, B. Bell, K.S.Kuo: Array Databases: Concepts, Standards, Implementations. Research Data Alliance (RDA) Working Group Report, May 2018, dx.doi.org/10.15497/RDA00024

[3] P. Baumann, V. Merticariu: On the Efficient Evaluation of Array Joins. Proc. IEEE Big Data Conference Workshop Big Data in the Geo Sciences, Santa Clara, US, October 2015

[4] P. Baumann: The Datacube Manifesto. http://earthserver.eu/tech/datacubemanifesto, seen 2017-05-28

[5] K. Boulil, S. Bimonte, F. Pinet: Conceptual model for spatial data cubes: A UML profile and its automatic implementation. Computer Standards \& Interfaces 38(2015), pp. 113-132

[6] A. Dumitru, V. Merticariu, P. Baumann: Array Database Scalability: Intercontinental Queries on Petabyte Datasets (system demonstration). Proc. 28th Intl. Conf. on Scientific and Statistical Database Management (SSDBM), Budapest, Hungary, July 18 - 20, 2016

[7] P. Furtado er al.: Storage of Multidimensional Arrays Based on Arbitrary Tiling. ICDE'99, March 23-26, 1999, Sydney, Australia

[8] Google: Earth Engine. https://developers.google.com/earth-engine/, seen on 2018-06-10

[9] N.n.: Matlab. https://www.mathworks.com/products/matlab.html, seen 2018-010

[10] N.n.: PostGIS Raster. http://postgis.net/docs/manual-2.1/RT_reference.html, seen 2018-06-10

[11] N.n.: SciDB. https://www.paradigm4.com, seen 2018-06-10

[12] N.n.: Open Data Cube. https://ac.els-cdn.com/S0034425717301086/1-s2.0S0034425717301086-main.pdf, seen n 2018-06-10

[13] N.n.: GeoTrellis. http://geotrellis.io/, seen on 2018-06-10

[14] N.n: xarray. http://xarray.pydata.org, seen on 2ß018-06-10

[15] N.n: Ophidia. http://ophidia.cmcc.it/, seen on 2018-06-10

[16] OGC Web Coverage Processing Service (WCPS) Interface Language, version 1.0, OGC document 08-068r2

[17] OGC: Coverage Implementation Schema, version 2.1, OGC document 09-146r3

[18] OGC: OGC Spatio-Temporal Coverage / Datacube Standards. http://myogc.org/go/coveragesDWG, seen 2018-06-10

[19] OGC: Web Coverage Service (WCS) Core, version 2.1, OGC document 09$110 \mathrm{r} 5$

[20] rasdaman: rasdaman: Agile Datacube Analytics. ACM SIGSPATIAL 2018 (submitted)

[21] Research Data Alliance: Array Databases: Concepts, Standards, Implementations. Research Data Alliance (RDA) Working Group Report, May 2018, dx.doi.org/10.15497/RDA00024

[22] P. Strobl et al: The Six Faces of The Datacube. Proc. Conf. on Big Data from Space (BiDS'17), 28-30 November 2017, Toulouse, France

[23] Kohler, K., P. Baumann: Diagnosis in the field - Big-data based determination of causes for satellite-image-derived and site-specific variations. Proc. Innovation days of the Federal Office for Agricultural and Food, Bonn, Germany, October 23-24, 2018 